Original Research

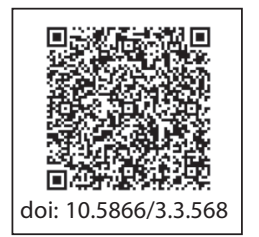

\title{
Prediction of Mesio-distal width of Canines and premolars in a sample of north Indian population
}

\author{
Pooja Ahluwalia', Sunaina Jodhka², Abi M. Thomas ${ }^{3}$
}

\section{Sr. Lecturer ${ }^{1}$}

Dept. Of Pedodontics and Preventive Dentistry

Gian Sagar Dental College \& Hospital,

Ram Nagar, Banur

Patiala.

Sr.Lecturer ${ }^{2}$

Professor, Principal and Head Of Dept ${ }^{3}$

Dept. Of Pedodontics and Preventive Dentistry

Baba Jaswant Singh Dental College \& Hospital, Ludhiana-141008.

\section{Article Info}

Received: April 13, 2011

Review Completed: May, 14, 2011

Accepted: June, 16, 2011

Available Online: October, 2011

() NAD, 2011 - All rights reserved

\begin{abstract}
:
Background: This study was done to evaluate the applicability of the Tanaka-Johnston method of prediction of mesiodistal widths of permanent canines and premolars in North Indian population.

Methods: 202 dental casts were obtained and mesiodistal widths of permanent lower incisors, canines and premolars were recorded using vernier calipers to nearest of $0.02 \mathrm{~mm}$. The predicted values of mesiodistal widths of permanent canines and premolars were obtained using Tanaka Johnston method. These values were put to statistical evaluation using Student't' test and Pearson's coefficient of correlation.

Results: The results revealed that the differences between the actual and predicted values of permanent canines and premolars were statistically significant in maxilla and mandible in both sexes $(p<0.05)$.

Conclusion: The present study suggests that Tanaka Johnston mixed dentition analysis overpredicted the mesiodistal dimension of unerupted premolars and canines in both male and female subjects and tooth dimension show sexual dimorphism with males having larger mesiodistal tooth dimensions than females
\end{abstract}

Key words: Tanaka Johnston analysis, Permanent canines, Premolars.

\section{INTRODUCTION}

Prediction of space required for unerupted canines and premolars is an important aid for treatment planning in interceptive orthodontics. This entails determination of tooth size to be done prior to eruption of canines and premolars by mixed dentition space analysis. Mixed dentition analysis is an important criterion in determining whether the treatment plan is going to involve serial extraction, guidance of eruption, space maintenance, space regaining or just periodic observation of patient. ${ }^{1} \mathrm{~A}$ simplified analysis proposed by Tanaka Johnston ${ }^{2}$ comes handy for chair side evaluation. Original Tanaka Johnston analysis was done on population of north European descent. ${ }^{3}$ It has been shown by Lavelle CLB (1972) that variations in tooth sizes occur based upon ethnicity of a particular population group and within the population, sexual dimorphism

Email for correspondence:

drpoojaahluwalia@yahoo.com; drsunaina79@yahoo.com.sg

abithomas@gmail.com with respect to tooth size prevails. ${ }^{4}$ Thus the accuracy of these predictive methods is questionable when applied to the Indian population.

Ludhiana ${ }^{5}$ in Punjab, being an industrial hub attracts people from all over India resulting in a cultural intermingling. Thus a sample population of Ludhiana will provide a good overview of the Indian population for testing of applicability of Tanaka Johnston analysis.

Very few studies in literature have been cited, addressing the applicability of using the standard Tanaka- Johnston equations for Indian population. Therefore, the objectives of this investigation were to examine the applicability of the Tanaka-Johnston method of prediction in a different population and to develop a standard prediction formula, by regression analysis, to be used for this specific population.

\section{MATERIALS AND METHODS}

The data were randomly collected from dental study casts obtained from the Dept. of Orthodontics, 
Christian Dental College, Ludhiana, Punjab, of 202 children of either sex, aged 12-18 yrs with full complement of dentition erupted (barring the second and third molars). Subjects were Indian born and of North -Indian ancestry and of Punjabi origin. All were permanent residents of Ludhiana, Punjab.

Dental casts were of high quality and free of distortion. To facilitate accurate measurements, only the casts with minor malocclusions were included such as minor crowding, rotation or diastema. Casts having teeth with obvious rotations, signs of attrition, tooth material loss due to caries, proximal wear or erosion, dental malformations, congenitally missing, impacted, extracted, supernumerary, geminated or supplemental teeth were excluded. The sample population fitting these criteria comprised of 85 male subjects and 116 female subjects.

Measurements were made with finely pointed Vernier calipers and read to the nearest $0.02 \mathrm{~mm}$. The greatest distance between contact points on the proximal surfaces of each tooth were measured with the calipers parallel to the occlusal plane. The predicted width of the canine, first premolar and second premolar, per quadrant, was calculated by measuring the sum of lower incisors and using the formula given by Tanaka- Johnston;

$\mathrm{Y}=\mathrm{I} / 2+10.5$, for lower arch.

$Y=I / 2+11$, for upper arch. (I is the sum of incisors and $Y$ is the predicted with of sum of canine and premolars, on one side of the arch)

The actual widths of the upper and lower canines and premolars were calculated for the left and right sides and their mean value was added to deduce the actual combined width of canines and premolars as the average difference between the right and the left sides did not differ statistically among the teeth in the upper and lower arch, as seen by a preliminary analysis.

By using this data, regression equations were formulated for the prediction of tooth size in Punjabi population. The least square regression equations were calculated as follows;

$\mathrm{Y}=\mathrm{A}+\mathrm{B}(\mathrm{X})$, where $\mathrm{Y}$ is the predicted size of canines and premolars in one quadrant in $\mathrm{mm}$ and $X$, is the measure width of lower permanent mandibular incisors in $\mathrm{mm}$. $(\mathrm{A}+\mathrm{B})$, are the constants to be derived.

\section{RESULTS}

Table I-Descriptive statistics for all subjects ( $\mathrm{mm}$ )

\begin{tabular}{lcccc} 
Sex & Segment & Mean $(\mathbf{m m})$ & Subjects & Standard Deviation \\
\hline Male & Md 2-2 & 23.82 & 85 & \pm 1.70 \\
\hline Mxp3-5 & 22.91 & 85 & \pm 0.85 \\
\hline Mxa3-5 & 22.41 & 85 & \pm 1.11 \\
\hline Mdp3-5 & 22.41 & 85 & \pm 0.85 \\
\hline Mda3-5 & 22.08 & 85 & \pm 0.98 \\
\hline Md2-2 & 23.06 & 116 & \pm 1.48 \\
\hline Mxp3-5 & 22.53 & 116 & \pm 0.74 \\
\hline & Mxa3-5 & 21.77 & 116 & \pm 1.27 \\
\hline & Mdp3-5 & 22.03 & 116 & \pm 1.31
\end{tabular}

Md2-2- Sum of mesiodistal widths of lower permanent incisors

Mxp3-5 -Combined mesiodistal width of maxillary canines and premolars per quadrant as predicted by Tanaka Johnston Mixed dentition analysis (T-J MDA)

Mxa3-5 -Actual combined mesiodistal width of maxillary canines and premolars per quadrant as measured on plaster cast.
Mdp3-5 - Combined mesiodistal width of mandibular canines and premolars per quadrant as predicted by Tanaka Johnston Mixed dentition analysis (T-J MDA)

Mda3-5 - Actual combined mesiodistal width of maxillary canines and premolars per quadrant as measured on plaster cast.

This table represents the mean mesiodistal combined widths of lower incisors and actual \&predicted sum of mesiodistal widths of canines\& premolars. 
Table II -Comparison of mesiodistal width of sum of incisors and actual combined widths of canines and premolars in male and female subjects

\begin{tabular}{ccccc} 
Tooth group & Sex & Sum of mesiodistal width $(\mathbf{m m})$ & t- value & p-value \\
\cline { 2 - 3 } Md2-2 & M & 23.82 & 2.34 & 0.021 \\
\cline { 2 - 3 } & $\mathrm{F}$ & 23.06 & & \\
\hline Mxa3-5 & $\mathrm{M}$ & 22.41 & 2.48 & 0.015 \\
\cline { 2 - 3 } & $\mathrm{F}$ & 21.77 & & \\
\hline Mda3-5 & $\mathrm{M}$ & 22.08 & 3.77 & 0.001
\end{tabular}

This table represents the mean differences between the mesiodistal widths of lower incisors, combined widths of canines and premolars in maxilla and mandible in both the sexes. Student- ' $t$ ' test was applied to evaluate whether these differences were statistically significant. The results show that there was significant difference between mesiodistal widths of teeth under evaluation in study as the $p$ - value for all the groups tested in the table is less than 0.05 .

Table III-Correlation Coefficient (Paired sample test)

\begin{tabular}{clccc} 
Sex & Pairs of tooth groups & Sample size(N) & Correlation (r) & Significance (P value) \\
Males & Md2-2 - Mxa3-5 & 85 & 0.51 & 0.002 \\
\hline & Md2-2 - Mda3-5 & 85 & 0.54 & 0.001 \\
\hline \multirow{2}{*}{ Females $3-5-$ Mxa3-5 } & 85 & 0.51 & 0.002 \\
\hline \multirow{2}{*}{ Mdp3-5 - Mdp3-5 $2-$ Mxa3-5 } & 85 & 0.54 & 0.001 \\
\hline & Md2-2 - Mda3-5 & 116 & 0.43 & 0.000 \\
\hline & Mxp 3-5 - Mxa3-5 & 116 & 0.39 & 0.001 \\
\hline
\end{tabular}

This table represents the relation between sum of lower incisors \& actual sum of mesiodistal widths of canines and premolars and actual \& predicted values of combined mesiodistal widths of canines and premolars, seen in maxilla and mandible for both the sexes. Pearson's coefficient of correlation was equated and results revealed that there was significant positive correlation between the values equated, in both males and females $(p<0.05)$.

Table IV- Paired student's ' $t$ ' test to evaluate difference in measured and predicted combined widths of canines and premolars

\begin{tabular}{llcccc} 
Sex & Pairs of groups & Mean difference & Standard deviation & t-value & p-value \\
\hline \multirow{2}{*}{ Males } & Mxp3-5 - Mxa3-5 & 0.50 & \pm 0.99 & 2.98 & 0.005 \\
\hline \multirow{2}{*}{ Femlaes } & Mdp3-5 - Mda3-5 & 0.33 & \pm 0.88 & 2.18 & 0.036 \\
\hline & Mxp3-5 - Mxa3-5 & 0.75 & \pm 1.17 & 5.22 & 0.000 \\
\hline
\end{tabular}

This table represents the mean difference between the actual and predicted sum of mesiodistal widths of canines and premolars. A Paired student ' $t$ ' test was applied to evaluate if these differences were statistically significant. The results revealed that the differences between the actual and predicted values were statistically significant in maxilla and mandible in both sexes $(p<0.05)$. 
New formulae using Regression equation

Males: Maxilla-14.439+Md2-2×0.336;

Mandible-14.576+Md2-2×0.315

Females: Maxilla-13.288+Md2-2×0.368;

Mandible-13.189+Md2-2×0.344

\section{Discussion}

The study was undertaken to evaluate the applicability of TJ MDS analysis in predicting combined mesiodistal widths in a sample north Indian population. Several studies have been carried out in this regard in different regions since the sex and race influenced variations in tooth dimensions are well known. Regression based MDS analysis specific to race and sex of the individual is evident in studies by Diagne F \& colleagues (2003), ${ }^{6}$ Jaroonthan J\& Godfrey K(2000), ${ }^{7}$ Yuen KK \& colleagues $(1995)^{8}$, Frankel HH \& Benz EM (1986) ${ }^{9}$ and Schirmer UR \&Wiltshire WA(1997). ${ }^{10}$

The mean values for teeth being evaluated in this study were found to greater in males as compared to females (Table1). The paired t-test, revealed that this difference was statistically significant (Table 2). Such a sexual dimorphism in tooth sizes is a fact well documented in humans and primates in previous investigations (Garn et al., 1967; Garn et al., 1977; Scott \& Turner, 1997; Kondo et al., 1998; Kondo \& Townsend; Kondo et al.,2005; Teschler-

Nicola, 1992; Wood et al., 1991). ${ }^{11}$ Thus, there is a need for separate formulae for males and females unlike the original equation given by Tanaka Johnston which used the same formula for both the sexes.

The results of the study also conclusively proved that the method of evaluation of the combined width of unerupted canines and premolars could be strongly correlated with the sum of the mesiodistal width of the lower permanent incisors (Table3). A good correlation between the actual and predicted values was found but the mean difference between actual and predicted combined widths of canines and premolars in males and females was found to be statistically significant with greater deviation from the actual values in mandibular arch in females (Table 4). This being caused by the fact that TJ MDS analysis overpredicts the combined tooth sizes and that the teeth are smaller in females as compared to males. The mean difference between actual and predicted combined widths of canines and premolars was found to be $1 \mathrm{~mm}$ in maxilla and $0.6 \mathrm{~mm}$ in mandible in males, $1.42 \mathrm{~mm}$ in maxilla and $1.80 \mathrm{~mm}$ in mandible in females.
So new regression equation was formed pertaining to the sample population keeping into view that a sexual dimorphism exists influencing tooth sizes. This would decrease the error in predicting tooth sizes, hence, preventing unnecessary sacrifice of teeth especially in females. This would also serve as a speedy chair side diagnostic tool for space analysis and treatment planning where the availability of prediction charts (as in Moyers analysis and Hixon \& Oldfather analysis) might not be feasible.

This proposed method of prediction however must be tested in a larger sample to confirm its applicability and consistency.

\section{Conclusion}

1. Tanaka Johnston mixed dentition analysis overpredicted the mesiodistal dimension of unerupted premolars and canines in both male and female subjects.

2. Tooth dimension show sexual dimorphism with males having larger mesiodistal tooth dimensions than females

\section{References}

1. Bishara SE, Staley RN.Mixed dentition mandibular arch length analysis: A step by step approach using Hixon-Oldfather prediction method. Am J Orthod Dentofacial Orthop. 1984.

2. Tanaka MM, Johnston LE. The prediction of the size of unerupted canines and premolars in a contemporary orthodontic population.JADA.1974; 88:798-801.

3. Irwin RD, Herold JS, Richardson A. Mixed dentition analysis: a review of methods and their accuracy.Inter J Pedatr Dent.1995;5:137-42.

4. Lavelle CLB. Maxillary and mandibular tooth size in different racial groups and in different occlusal categories. Am J Orthod.1972; 61(1):29-36.

5. http://en.wikipedia.org/wiki/Ludhiana.

6. Diagne F, Diop-Ba K, Ngom Pl, Mbow K. Mixed dentition analysis in a sengalese population:elaboration of prediction tables. Am J Orthod Dentofacial Orthop.2003;124:178-183.

7. Jaroontham J, Godfrey K. Mixed dentition space analysis in a thai population. Eur J Orthod.2000;22: 127-134.

8. Yuen KK, Tang EL, So LL. Mixed dentition analysis for Hong Kong Chinese. Angle Orthod.1995;68:21-28.

9. Frankel HH, Benz EM. Mixed dentition analysis for black Americans.Peaditr Dent1986;8: 226-230.

10. Schirmer UR, Wiltshire WA. Orthodontic probability tables for black patients of African descent: mixed dentition analysis. Am J Orthod Dentofacial Orthop.1997;112:545-551.

11. Suazo GI, Cantin LM, Lopez FB, Sandoval MC, Torress MS, Gajardo RP, Gajardo RM.

Sexual dimorphism in mesiodistal and bucolingual tooth dimensions in Chilean people. Int. J. Morphol.2008; 26(3):609-614. 